\title{
Microlensing Maps for the Galactic Bulge
}

\author{
N.W. Evans \\ Room 2-367, Department of Mathematics, Massachusetts Institute of Technology, \\ Cambridge, MA 02139, USA
}

Received — 


\begin{abstract}
Microlensing maps - that is, contours of equal numbers of events per $10^{6}$ source stars - are provided for the inner Galaxy under two alternative hypotheses : (1) the bulge is an oblate axisymmetric spheroid or (2) the bulge is a prolate bar. Oblate spheroids yield a total of $\sim 12$ events per year per $10^{6}$ stars at Baade's Window ( $\sim 15$ events if the disk is maximal). The event rate is slightly lower for prolate bars viewed at $\sim 45^{\circ}$ and the maps have a characteristic asymmetry between positive and negative longitudes. Prolate bars can yield mild amplifications of the event rate if viewed almost down the long axis. The disk provides the dominant lensing population on the bulge major axis for $|\ell| \gtrsim 6^{\circ}$. Measurements of the rate at major axis windows can test for disk dark matter or maximal disk models.
\end{abstract}

Subject headings: dark matter - Galaxy: stellar content - Galaxy: structure gravitational lensing 


\section{INTRODUCTION}

The startling reports of enhanced rates of microlensing towards the Galactic bulge (Alcock et al. 1994, Udalski et al. 1994a, Udalski et al. 1994b) confront us with immediate challenges. Are the observations consistent with the known populations of deflectors? Do the microlenses lie in the Galactic disk or in the bulge or even - as recently suggested by Paczyński et al. (1994) - in a bar? The aim of this Letter is to suggest observational tests that can provide answers to these questions.

\section{BULGE, BAR AND THICK DISC MODELS}

Let us first introduce models for the components of the inner Galaxy. A simple, though idealised, oblate axisymmetric bulge model is provided by Evans \& de Zeeuw (1994, hereafter EZ). Their starting point is Becklin \& Neugebauer's (1968) observation that the emissivity profile of the inner bulge is scale-free and falls like $\rho \sim r^{-1.8}$. So, a good representation of the density profile of the cusp is provided by a scale-free oblate power-law model (Evans 1994, hereafter E94). The density of the model is

$$
\rho \sim 5.9 \times 10^{8} \frac{R^{2}+0.87 z^{2}}{\left(R^{2}+1.23 z^{2}\right)^{1.9}} \mathrm{M}_{\odot} \mathrm{kpc}^{-3}
$$

where $R$ is the cylindrical polar radius and $z$ the height above the galactic plane in kiloparsec. The model has an apparent axis ratio $a_{2} / a_{1}$ of 0.75 (Habing 1988) and a line of sight velocity dispersion at Baade's Window of $119 \mathrm{kms}^{-1}$ (c.f. Kent 1992). Outside of the inner kiloparsec, the luminosity density of the galactic bulge falls like $r^{-3.5}$. We do not model this in detail, but truncate (1) at some distance $D_{\text {cut-off }}$, beyond which the density is assumed to vanish. We choose $D_{\text {cut-off }}$ to be $3 \mathrm{kpc}$ so that the total bulge mass is 
$\sim 1.9 \times 10^{10} \mathrm{M}_{\odot}$. The advantage of EZ's bulge over Kent's (1992) hydrodynamical model is that the self-consistent distribution of proper motions for the former is explicitly available (see EZ, Appendix B).

An alternative viewpoint is to regard the bulge as a prolate bar viewed somewhat broad-side on (de Vaucouleurs 1964). A number of investigators have concluded that the evidence from neutral and ionised gas motions (Binney et al. 1991, Blitz \& Spergel 1991) and star-counts (Weinberg 1992, Stanek et al. 1994) suggests that the Galaxy is barred. We shall use a prolate power-law model (E94) to represent the bar (c.f., Binney et al. 1991)

$$
\rho \sim 3.7 \times 10^{8} \frac{x^{2}+0.89\left(y^{2}+z^{2}\right)}{\left(0.67 x^{2}+y^{2}+z^{2}\right)^{1.9}} \mathrm{M}_{\odot} \mathrm{kpc}^{-3}
$$

Here, the $x$-axis defines the long axis of the bar, which is oriented at an angle $\theta$ to the line joining the Sun to the Galactic Center and the model is truncated at $D_{\text {cut-off }}=3 \mathrm{kpc}$. Our bulge (1) and bar (2) models have the same total mass and roughly the same apparent flattening. Although all investigators agree that the near-side of the bar lies at positive Galactic longitudes $(\ell>0)$, there is no concensus over the angle $\theta$ at which we view the major axis. Note that EZ's distribution of proper motions for the power-law models assume the figure is fixed in inertial space. This is a fair approximation if the pattern speed of the bar is small (c.f. Blitz \& Spergel 1991).

Lastly, we need a model of the Galactic disk, whose density we assume to be exponentially declining on spheroidal surfaces

$$
\rho \sim 3.9 \times 10^{8} \exp \left(-0.29 \sqrt{R^{2}+144 z^{2}}\right) \mathrm{M}_{\odot} \mathrm{kpc}^{-3} .
$$

The axis ratio $a_{2} / a_{1}$ is 0.083 , as suggested by Bahcall \& Soneira's (1980) analysis. The scale-length of the disk is $3.5 \mathrm{kpc}$. The normalisation is chosen to recover the local column density of $\sim 50 \mathrm{M}_{\odot} \mathrm{pc}^{-2}$ (Gould 1990). It is sometimes suggested that this data may be misleadingly low. An upper limit is provided by 'maximal disk' models, in which disk 
matter provides almost all the local centrifugal balance (e.g., Alcock et al. 1994). If the disk is maximal, then the density (3) is roughly doubled. Self-consistent velocity distributions for thick disks are not known - but this is not too serious as the random motions are much smaller than the systemic rotation of $\sim 200 \mathrm{kms}^{-1}$.

\section{MICROLENSING MAPS}

The contributions of different deflecting populations can be distinguished by the variation in microlensing rate as a function of Galactic longitude and latitude. A simple way to picture this is provided by microlensing maps - that is, contours of equal numbers of events per $10^{6}$ source stars. The maps are useful because they are lots of windows of low extinction towards the bulge. Even along heavily obscured lines of sight, the main effect of interstellar extinction is to reduce the number of detectable source stars. This just alters the overall normalisation of the rate.

The microlensing rate $\Gamma$ is the reciprocal of the time between events averaged over all possible lensing configurations. So, $\Gamma$ depends not only on the densities but also the distributions of masses and proper motions of the sources and the deflectors. Kiraga \& Paczyński (1994, hereafter KP) introduced a refinement by modulating the density of the sources by a factor of $D_{\mathrm{s}}^{2 \beta}$, where $D_{\mathrm{s}}$ is the distance of the source from the sun. This is a crude way of modelling the tendency to observe fewer stars at the far side of the bulge. The value $\beta=-1$ is reasonably close to what is observed (Depoy et al. 1993). To construct microlensing maps, the rate is calculated at any Galactic longitude and latitude $(\ell, b)$ using eq. (11) of KP. The tangential velocity components of the bulge or bar stars are randomly drawn from the distributions of proper motions of the power-law models (EZ). The distribution of stellar masses is taken as a Gaussian in $\log _{10} M$ for masses greater than 
$0.35 \mathrm{M}_{\odot}$ and flat below $0.35 \mathrm{M}_{\odot}$ to a lower cut-off of $0.01 \mathrm{M}_{\odot}$ (Kroupa, Tout \& Gilmore 1990).

Figure 1 shows a map for the bulge (1) and disk (3) model of the inner Galaxy. The contributions from the different deflector populations have been separated. The full (broken) contours delineate the number of events per year per $10^{6}$ source stars caused by deflectors in the bulge (disk). If the disk is maximal, then the share from the disk lenses is nearly doubled. The optical depth at Baade's Window $\left(\ell=1.0^{\circ}, b=-3.9^{\circ}\right)$ is $6.3 \times 10^{-7}$ for microlensing by bulge lenses, $5.0 \times 10^{-7}$ for disk lenses. The frequency of events is $\sim 12$ per year per $10^{6}$ stars, of which $\sim 70 \%$ are caused by bulge lenses (c.f. KP). This rises to $\sim 15$ events if the disk is maximal. The unbroken logarithmic contours in the map are almost equally spaced, indicating that microlensing by bulge deflectors declines roughly exponentially with projected distance from the Galactic Center. The broken contours are almost horizontal, confirming KP's insight that the influence of disk lenses is nearly independent of $\ell$. All this suggests a number of observational tests. First, the disk lenses quickly become more important than the bulge lenses on the projected major axis. At the clear window at $\left(\ell=12.0^{\circ}, b=3.0^{\circ}\right)$, maximal disc models yield $\sim 11$ events per year per $10^{6}$ stars, of which more than $70 \%$ are caused by disk lenses. A large event rate measured at major axis windows with $|\ell| \gtrsim 6^{\circ}$ is an unambiguous signature of disk deflectors. A second test is to compare the rate in bulge fields at roughly the same latitude. There are clear windows at $\left(\ell=1.0^{\circ}, b=-3.9^{\circ}\right)$ and $\left(\ell=5.5^{\circ}, b=-3.5^{\circ}\right)$, which are candidates for the application of this test. The contribution of bulge lenses to the rate varies by $\sim 30 \%$, while the contribution of disk lenses remains unchanged. Variation in the rate at locations with the same latitude provides evidence that the deflector population is in the bulge. Inset into Figure 1 are the expected frequencies of event timescales $t_{0}$ for the bulge (full lines) and disk (dashed lines) lenses at two fields currently undergoing intense scrutiny - one is at Baade's window, the other is closer to the Galactic Center $\left(\ell=2.3^{\circ}, b=-2.65^{\circ}\right)$. As 
Paczyński (1991) and Griest et al. (1991) point out, the average timescale is longer for deflectors in the disk. Notice that this occurs mainly because the dashed distributions have larger tails towards the longer events. At Baade's Window, the peak timescale is $\sim 15$ days, irrespective of whether the deflector is a bulge or disk star. This is longer than that found by KP of $\sim 10$ days. Now, the efficiency of the microlensing experiments cuts off sharply at short timescales. If the distribution is peaked at longer timescales, this may mean that the efficiency has been underestimated, leading to values of the optical depth derived from the experimental results that are too high.

Figure 2 shows a map for the bar (full lines) and disk (broken lines). The position angle of the bar's major axis $\theta$ is chosen as $45^{\circ}$ (c.f. Blitz \& Spergel 1991). The optical depth at Baade's Window is $5.3 \times 10^{-7}$ for bar lenses, $5.1 \times 10^{-7}$ for disk lenses. The rate is $\sim 10$ events per year per $10^{6}$ source stars. The map is asymmetric. For bar lenses, the number of events is higher at negative longitudes as compared to positive longitudes. This is because the near-side of the bar lies in the first Galactic quadrant, and so lines of sight to detectable stars for $\ell>0$ are on average shorter and pass through less of the dense inner bar than for $\ell<0$. This is analogous to the phenomenon that Crotts (1992) and Gould (1993) noticed for the optical depth of the inclined disks of M31 and the Large Magellanic Cloud. For disk lenses, the reverse is true. The rate is slightly higher at positive longitudes. This is because the rate is averaged over the detectable stars along the line of sight, with nearer sources carrying more weight than more distant sources. For $\ell>0$, the sources are on average closer, thereby amplifying the rate as compared to $\ell<0$. This effect is model-dependent and diminishes with increasing $\beta$. Udalski et al. (1994b) are already scanning the windows at $\left(\ell=-5.0^{\circ}, b=-3.5^{\circ}\right)$ and $\left(\ell=5.5^{\circ}, b=-3.5^{\circ}\right)$ for possible evidence of the asymmetric microlensing signal of a bar. In our model, $\sim 11$ events per year per $10^{6}$ sources are expected at the former location, $\sim 7$ at the latter. Now, Blitz \& Spergel's (1991) estimate of the position angle of the bar's major axis $\theta \sim 45 \pm 20^{\circ}$ is in 
broad agreement with Weinberg's (1992) conclusion of $\theta \sim 36 \pm 10$ based on an analysis of the IRAS 2 micron sources. But, both results are in apparent conflict with Binney et al. (1991), who claim $\theta \sim 16 \pm 2^{\circ}$ from an ingenious analysis of the kinematics of the Galactic Center gas. How does the microlensing rate change as the viewing angle of the bar is varied? The number of events at Baade's Window is plotted against $\theta$ and inset into Figure 2. As we view the bar more nearly down its major axis, the event rate slowly increases to $\sim 13$ events per year per $10^{6}$ stars $(\sim 16$ events if the disk is also maximal). This is a lower limit of the expected amplification. Our prolate bar (2) looks round when $\theta \sim 0^{\circ}$ and so does not properly represent the flattening of the bulge. The neglect of the figure rotation and internal streaming motions of the bar might also be expected to lead to an under-estimate of the true rate. Nonetheless, the effect of distending material along the line of sight seemingly causes only a mild enhancement. Even if the bar is viewed at $\theta \sim 0^{\circ}$, the number of events at Baade's Window is only just greater than for our oblate axisymmetric model (1). Although it is possible that other bar models exist with greater amplification, the case for interpreting the high optical depths reported by Alcock et al. (1994) and Udalski et al. (1994b) as evidence for a bar remains unproven. Also inset into figure 2 is the asymmetric signal - that is, the percentage fractional difference in the rates at the windows at $\left(\ell=-5.0^{\circ}, b=-3.5^{\circ}\right)$ and at $\left(\ell=5.5^{\circ}, b=-3.5^{\circ}\right)$ - plotted against viewing angle $\theta$. If the bar is seen edge- on or pole-on, this asymmetric signal almost vanishes (not quite, because the windows are not exactly mirror images in $\ell=0$ ). But, the asymmetry of the microlensing maps quickly becomes evident as the orientation of the bar is varied. Even if the bar is viewed at $\sim 16^{\circ}$, the variation in the rates at $\left(\ell=-5.0^{\circ}, b=-3.5^{\circ}\right)$ and at $\left(\ell=5.5^{\circ}, b=-3.5^{\circ}\right)$ is still large enough to be detectable.

\section{CONCLUSIONS}


Microlensing maps describe the variation in event rate as a function of Galactic longitude and latitude. This is valuable in untangling the contributions of the disk and bulge lenses. We find:

(1) Oblate axisymmetric models of the bulge yield a total of $\sim 12$ events per year per $10^{6}$ stars at Baade's Window ( 15 events if the disk is maximal). Prolate bars can give mild enhancements of the rate if viewed at $\theta \sim 0^{\circ}$. Slowly rotating prolate bars with inclination angles of $\sim 45^{\circ}$ have lower rates than oblate models. Unless the bar is seen edge- on or pole-on, the microlensing maps are asymmetric with larger numbers of events expected at negative longitudes as compared to positive longitudes. Overall, the evidence for a bar in the inner Galaxy is strong. So, can the high optical depths reported in Alcock et al. (1994) and Udalski et al. (1994) be explained if the microlenses lie in a bar? Our study of a prolate bar suggests that this is not the whole story, but further modelling with triaxial and swiftly rotating bars is required for a final answer. The efficiency of the microlensing experiments depends partly on the distribution of timescales of events. At Baade's Window, we find this to peak at $\sim 15$ days - longer than previous studies (KP). This may mean the efficiency is being underestimated, accounting for some of the discrepancy between theoretical and experimental results.

(2) On the major axis for $|\ell| \gtrsim 6.0^{\circ}$, the microlensing rate is dominated by contributions from the disk deflectors. So, measurements of the rate at major axis windows test directly for disk dark matter or maximal disk models. For example, maximal disk models provide $\sim 11$ events per year per $10^{6}$ sources at the clear window at $\left(\ell=12.0^{\circ}, b=3.0^{\circ}\right)$, of which over $70 \%$ are caused by the disk lenses.

NWE is particularly indebted to Kim Griest, Kris Stanek and the referee for numerous helpful comments and suggestions. Useful discussions with Charles Alcock, Dave Bennett, 
Kem Cook and Peter Quinn during a visit to Lawrence Livermore National Laboratory are also acknowledged. NWE is supported by the Lindemann Trust and the Royal Society. 


\section{REFERENCES}

Alcock C., et al. 1994, ApJ, in press

Bahcall J.N., \& Soneira R. 1980, ApJS, 44, 73

Becklin E.E., \& Neugebauer G. 1968, ApJ, 151, 145

Binney J.J., Gerhard O.E., Stark A.A., Bally J., \& Uchida K.I. 1991, MNRAS, 252, 210

Blitz L., \& Spergel D. 1991, ApJ, 379, 631

Crotts A. 1992, ApJ, 399, L43

de Vaucouleurs G. 1964, In IAU Symposium 20, The Galaxy and the Magellanic Clouds, eds Kerr F.J., \& Rodgers A.W., (Australian Academy of Science, Canberra), p. 195

Depoy D.L., Terndrup D.M., Frogel J.A., Atwood B., \& Blum R. 1993, AJ, 105, 2121

Evans N.W. 1994, MNRAS, 267, 333 (E94)

Evans N.W., \& de Zeeuw, P.T. 1994, MNRAS, in press (EZ)

Gould A. 1990, MNRAS, 356, 302

Gould A. 1993, ApJ, 404, 451

Griest K., et al. 1991, ApJ, 372, L79

Habing H. 1988, A\&A, 200, 40

Kent S.M. 1992, ApJ, 387, 181

Kiraga M., \& Paczyński B. 1994, ApJ, 430, L101 (KP)

Kroupa P., Tout C., \& Gilmore G. 1990, MNRAS, 244, 76

Paczyński B. 1986, ApJ, 304, 1

Paczyński B. 1991, ApJ, 371, L63 
Paczyński B., et al. 1994, ApJL, submitted

Stanek K.Z., et al. 1994, ApJ, 429, L73

Udalski A., et al. 1994a, ApJ, 426, L69

Udalski A., et al. 1994b, Acta Astron., 44, 165

Weinberg M. 1992, ApJ, 384, 81 


\section{FIGURE CAPTIONS}

Fig. 1. - Logarithmic contours of equal numbers of microlensing events for the bulge (1) and disk (3) plotted in the plane of Galactic longitude $\ell$ and latitude $b$. Unbroken lines depict the contribution from the bulge lenses (respectively 20,10, 5 and 2.5 events per year per $10^{6}$ stars moving outwards from the centre). Broken lines show the contribution from disk lenses (respectively 5, 2.5 and 1.25 events). The insets show the distribution of events as a function of timescale $t_{0}$ for the bulge (unbroken lines) and disk (broken lines) deflectors at Baade's Window $\left(\ell=1.0, b=-3.9^{\circ}\right)$ and at $\left(\ell=2.3^{\circ}, b=-2.65^{\circ}\right)$.

Fig. 2.- Logarithmic contours of equal numbers of microlensing events for the bar (2) and disk (3). The position angle of the major axis of the bar $\theta$ is $45^{\circ}$. The unbroken lines are the contributions from the bar lenses, the broken lines are the contributions from the disk lenses. The inset (a) shows the variation of the microlensing rate at Baade's Window (measured in events per year per $10^{6}$ source stars) with the viewing angle of the bar $\theta$. The inset (b) shows the variation of the asymmetric signal - defined as the percentage fractional difference in the total rate at the clear windows at $\left(\ell=-5.0^{\circ}, b=-3.5^{\circ}\right)$ and $\left(\ell=5.5^{\circ}, b=-3.5^{\circ}\right)$ - with viewing angle $\theta$. In inset (b), we have not separated the contributions from bar and disk lenses. Of course, the contribution to the asymmetric signal from the disk lenses is almost negligible. 
This figure "fig1-1.png" is available in "png" format from: http://arxiv.org/ps/astro-ph/9409002v1 
This figure "fig1-2.png" is available in "png" format from: http://arxiv.org/ps/astro-ph/9409002v1 